\title{
La formación corporal en Psicomotricidad Observaciones de los parámetros de interacción corporal en la identidad de base
}

LA FORMAZIONE CORPOREA IN PSICOMOTRICITÁ

OSSERVAZIONE DEI PARAMETRI D'INTERAZIONE CORPOREA NELL'IDENTITÁ DI BASE

Dra. Stefania Pontrandolfo ${ }^{1}$ Lic. Psicom. Pabla Beatriz Ruiz Méndez ${ }^{2}$

${ }^{1}$ Antrolpóloga. Docente e investigadora en el Departamento de Filosofía, Pedagogía y Antropología. Universidad de Verona - Italia

${ }^{2}$ Psicomotricista, Máster Internacional en Psicomotricidad, Licenciada en Ciencias de la Educación, Actualmente ejerce la profesión en Verona - Italia 


\section{INTRODUCCIÓN}

Resulta interesante comprender cómo la psicomotricidad, considerando la persona en una óptica de integración entre los elementos funcionales, experienciales, emocionales e intelectuales, puede complementar su objeto de estudio con la Antropología Cultural, que hoy ocupa un espacio "fundamental" en el ámbito de los saberes en cuanto forma de vida. Estudiando la diversidad/autonomía de las culturas, se ha puesto a la luz, a través de la etnografía, los conocimientos entre las fusiones de cuerpo, cultura e identidad.

A continuación, presentar la importancia de la formación corporal como elemento indispensable en el crecimiento profesional del psicomotricista, específicamente en la formación de los estudiantes de la carrera de Psicomotricidad del CISERPP y de la UNIBERO. En esta última se aborda el "paradigma chamánica", un elemento trasversal en la cultura paraguaya, asumiendo que cada grupo humano produce culturas, normas y conocimientos, sobre las bases de datos y experiencias al menos parcialmente compartibles. Los valores compartibles de una comunidad introducen las dimensiones de la subjetividad y del intersubjetividad en el discurso de la construcción de conocimiento y conciencia.

Sucesivamente, las investigaciones realizadas hacen referencia a los datos que emergen de las observaciones de los parámetros de interacción corporal, en dos contextos diferentes a través de las técnicas corporales, "elementos indispensables" en nuestra profesión. Solo interiorizando y canalizando estas últimas en nuestro cuerpo, podemos sentir, vivir, compartir y abordar el lenguaje corporal de los demás (el paciente). El cuerpo es un espacio ideal, donde la puesta en acto de las técnicas corporales permite el desarrollo y la concienciación de la propia identidad, elemento indispensable en el trabajo de los psicomotricistas.

Por último, indagar sobre la valorización de la profesión de parte de los estudiantes psicomotricistas a través de la entrevista.

Palabras clave: psicomotricidad, cuerpo, formación corporal, cultura, identidad, paradigma.

\section{UNIBE REVISTA}




\section{INTRODUZIONE}

È interessante capire come la psicomotricità, considerando l'individuo in un'ottica di integrazione tra gli elementi funzionali, esperienziali, emozionali ed intellettivi, possa complementare il suo oggetto di studio con l'antropologia culturale, che oggi, occupa uno spazio "fondativo" nell'ambito dei saperi in quanto "forme di vita". Quindi, studiando le diversità/autonomie delle culture, ha messo in luce, attraverso l'etnografia, le conoscenze tra la fusione di corpo, cultura e identità.

Nei seguenti capitoli, accennerò l'importanza della formazione personale corporea come elemento indispensabile nella crescita dello psicomotricista, senza escludere finalità e programmi di formazione tanto in CISERPP $^{3}$ come in $\mathrm{UNIBERO}^{4}$. In quest'ultimo parlerò del "paradigma sciamanico", un elemento trasversale nella cultura paraguaiana, assumendo che ogni gruppo umano, produce cultura, norme e conoscenza sulla base di assunti ed esperienze almeno parzialmente condivisi. I valori condivisi da una "comunità" introducono le dimensioni della soggettività e dell' intersoggettività nel discorso sulla costruzione di conoscenza e consapevolezza.

Nei capitoli successivi, imposterò la mia ricerca sui dati che emergono dall'osservazione dei parametri d'interazione corporea, impostandola su due contesti differenti attraverso le tecniche corporee, "elementi indispensabili" nella nostra professione. Soltanto interiorizzando e canalizzando queste ultime nel nostro corpo, possiamo sentire, vivere, condividere e farci carico del linguaggio corporeo altrui (il paziente). Il corpo è uno spazio ideale, dove la messa in atto delle tecniche corporee permette lo sviluppo e la consapevolezza della propria identità, elemento indispensabile nel lavoro degli psicomotricisti.

Proprio per questo andrò ad indagare sulla valorizzazione della professione degli psicomotricisti attraverso l'intervista.

Parole chiave: psicomotricità, copro, formazione corporale, cultura, identità, paradigma.

${ }^{3}$ CISERPP, Centro Italiano Studi e Ricerche in Psicologia e Psicomotricità. Verona - Italia ${ }^{4}$ UNIBERO, Universidad Iberoamericana. Asunción - Paraguay. 


\section{PLANTEAMIENTO DEL PROBLEMA}

Sabemos que para ser psicomotricistas, es necesario poner en acto un proceso educativo basado en diferentes áreas: conocimientos teóricos, formaciones profesionales y formaciones personales. Esta formación es un proceso complejo de intercambios de contenidos y métodos para dar a conocer o hacer que el educando tome conciencia de los niveles funcionales, intelectuales, emocionales y comunicativos relacionales siempre mayores.

Esto significa que el educando psicomotricista debe adquirir, ser consciente y al mismo tiempo estar impregnado de conocimientos que caracterizan la propia profesión.

$\mathrm{Al}$ inicio la formación teórica se ha nutrido en conocimientos relativos a los de las áreas médicas y psicológicas; en particular en un profundo conocimiento del desarrollo evolutivo del niño. En cambio, en la actualidad, debemos considerar los aportes de la filosofía, antropología y sociología que dan énfasis al aspecto biopsicosocial del ser humano.

La formación profesional, implica la capacidad operativa relativa al saber hacer que comporta la adopción de un determinado procedimiento comportamental en donde se accionan las técnicas y metodologías adquiridas en el proceso formativo.

Un elemento que caracteriza la profesión del psicomotricista es seguramente la formación corporal, que se concretiza en la utilización del cuerpo y el movimiento. Es un proceso individual inserto en un proceso de grupo en donde cada sujeto dará forma a su aprendizaje según de la experiencia vivida. Es un espacio privilegiado de los estudiantes en donde viven situaciones que estimulan la búsqueda de concienciación y trasformación de sus potencialidades, expresivas y comunicativas en el plano psicomotriz.

Es interesante subrayar que cada cultura presenta un paradigma que es importante atender en la implementación de la formación corporal. El concepto de paradigma, en su sentido global, "indica una entera constelación de creencias, valores, técnicas compartibles por los miembros de una comunidad" (Kuhn, 1996, p.12). Es decir, un sistema sociocultural, socialmente compartible.

Sabemos que según la historia colonial de América Latina, y en particular del Paraguay, la formación formal es un producto cultural del Occidente que en la sociedad guaraní fue vivenciado por personas que, fuera de

314 UNIBE \begin{tabular}{l|l} 
REVISTA \\
CIENTIFIFICA
\end{tabular}


ella, viven procesos de construcción de la conciencia, de educación y socialización culturalmente diferente. En los procesos educativos de América Latina existen flujos de dos paradigmas que interaccionan entre sí implícitamente, es decir: el "paradigma chamánico" y el "paradigma occidental"; la primera índica "conjunto de modelos de la construcción socio-culturalmente guaraní, en cambio, la segunda índica el conjunto de modelos occidentales de crear el mundo" (Mastromattei 1999, p. 1).

En síntesis, este paradigma chamánico se traduce en la persona del paraguayo/a que tiene en el profundo de su ser, es decir en su karaku un modo propio de ser el ñande reko ${ }^{6}$. De consecuencia, en la vida cotidiana del paraguayo tiene mucho peso la raíz guaraní respecto del de los colonizadores.

Esta investigación se basa en un estudio explorativo-etnográfico, sobre las observaciones de los parámetros de interacción corporal en la identidad de base durante la formación personal de los educandos psicomotricistas.

Desde este contexto, se plantean las siguientes interrogantes:

1. Durante el proceso de formación corporal, en las observaciones de los parámetros de interacción corporal en la identidad de base, en los estudiantes de la UNIBERO. ¿Subsiste la influencia del paradigma chamánico? ¿Cómo emerge en los estudiantes durante el proceso formativo corporal?

2. Utilizando la misma actividad de expresión corporal con los estudiantes del CISERPP y de la UNIBERO en la correlación de los parámetros psicomotores evaluados, ¿Existen diferencias significativas entre ambos países (Paraguay e Italia)?

3. ¿Qué conocimientos presentan los alumnos de ambos países sobre la profesión? ¿Qué importancia presenta la formación corporal para los estudiantes?

4. Siendo la identidad parte integrante de la persona, ¿Qué datos emergen durante el proceso de formación corporal en la representación del diseño de la figura humana?

${ }^{5}$ Karaku, vocablo guaraní que significa médula.

${ }^{6}$ Ňande reko, expresión guaraní que significa nuestro modo de ser. 


\section{JUSTIFICACIÓN Y ALCANCE}

En la formación corporal, es importante entrar en contacto con la propia naturaleza, con las características de la personalidad, aprender a gestionar sentimientos y emociones, ir hacia el descubrimiento del poder personal, del yo auténtico, es decir, tomar conciencia del propio valor. La formación personal, al final de su recorrido, permite adquirir al educando psicomotricista mayor conocimiento de sus propias modalidades comunicativas, sean verbales y no verbales, en la relación consigo mismo, los objetos y los demás.

El proceso de cambio se da en la mayoría de los educandos a través de la relación con el otro que, inconscientemente, modifica la imagen corporal del sujeto. Sucesivamente, gracias a la formación corporal, esto se da conscientemente a través de dos visones: por una parte, una visión psicodinámica: capacidad, destrezas, habilidad, coordinación, etc. Por otra parte, una visión tónica: sentimientos, emociones y placeres.

El gran desafío hoy en día consiste en salvaguardar la cultura paraguaya, en un mundo cada vez mayormente globalizado, focalizando la atención en la educación y valorizando la identidad del país.

Desde el punto de vista de la psicomotricidad, hay mucho por hacer, donde se trabaja el cuerpo como mediador común de relaciones y de comunicaciones. Es importante focalizar la atención sobre la formación corporal de los futuros psicomotricistas en primera persona, ya que ellos/ as serán los especialistas del lenguaje corporal del otro.

El espacio formativo es un lugar donde viene revalorizada la persona como sujeto de relación consigo mismo y los demás. Donde se descubren las potencialidades y se aceptan las limitaciones; es decir, donde el cuerpo es un medio y un fin para expresar y valorizar la propia identidad implícita en las diferentes dimensiones de la persona: intelectiva, motora, afectiva, comunicativa-relacional.

El descubrimiento y la revalorización de la identidad de base son el fundamento de la psicomotricidad. El reconocimiento de la identidad de base se construye en la relación con el otro en un proceso de reconocimiento consigo mismo y con los demás, sobre las bases de la conciencia de un yo corporal en la continuidad temporal.

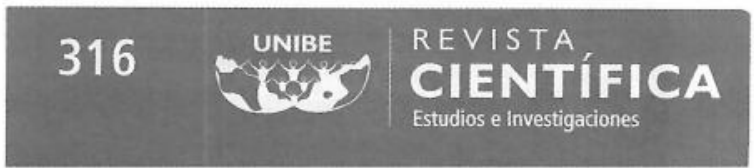




\section{OBJETIVOS}

\section{Objetivo General}

- Analizar si en las observaciones de los parámetros de interacción corporal emergen datos sobre la identidad de base durante el proceso de la formación corporal en psicomotricidad en dos países diferentes.

\section{Objetivos Específicos}

- Aplicar la psicomotricidad a través de la formación corporal como medio de exploración del ser humano que le permita el conocimiento de sí, de su entorno, así como del reconocimiento y fortalecimiento de su identidad.

- Reconocer la cultura como dato transversal en el desarrollo de la identidad de base.

- Confirmar la importancia de la psicomotricidad para la revalorización de la identidad en un mundo globalizado.

\section{Sujetos}

Los sujetos que participan en la investigación son estudiantes de psicomotricidad del CISERPP y algunos estudiantes y neopsicomotricistas de la UNIBERO que han frecuentado el curso de formación de la Licenciatura en Psicomotricidad según el programa establecido.

En la observación de los parámetros psicomotores, el análisis se ha efectuado sobre un total de 16 sujetos: 9 del CISERPP (identificados con la sigla c1, c2, c3, etc.) y 7 sujetos de la UNIBERO (identificados con la sigla i1, i2, i3, etc.)

Los sujetos del CISERPP fueron invitados a participar voluntariamente en la actividad de investigación, a través de una nota al responsable de la institución. En cambio, los sujetos de la UNIBERO fueron invitados a participar voluntariamente a la actividad de investigación a través de un encuentro realizada por $\mathrm{APP}^{7}$.

${ }^{7}$ APP, Asociación Paraguaya de Psicomotricidad. 


\section{Recursos materiales}

Seguidamente, serán elencados y presentados en anexo los materiales utilizados en el desarrollo de la investigación.

- Materiales bibliográficos: se refieren a los libros consultados específicamente aquellos de carácter etnográfico.

- Escala de programación de la actividad de formación corporal: la actividad desarrollada en el encuentro con los sujetos investigados presenta una intencionalidad para observar los parámetros de interacción corporal.

- Escala de observación de los parámetros de interacción corporal y filmación: es una escala elaborada con base en el Examen Psicomotor del Dr. Boscaini. "El objetivo del examen psicomotor no es exclusivamente, como en algunos casos, la revelación del déficit funcional sino más bien relevar el desorden de tipo cualitativo que se explica sólo al interno de la relación psicocorporal" (Boscaini, 2008, p. 3). La accesibilidad a los datos cualitativos permite observar en el plano psico-afectivo, tónico emocional, cognitivo, motriz, relacional, comportamental y comunicativo.

En la escala se completa por cada sujeto los datos observables a través del análisis de la filmación realizada durante el encuentro de formación corporal en psicomotricidad. Tratando de dar un puntaje objetivo a cada parámetro observable (desde 5 hasta 10 puntos), sin omitir algunos datos de órdenes cualitativos, datos relevantes en la labor del psicomotricista.

\begin{tabular}{|c|c|c|c|c|c|}
\hline $\begin{array}{c}\text { Evaluación } \\
\text { numérica }\end{array}$ & 5 & 6 & 7 & $8 / 9$ & $9 / 10$ \\
$\begin{array}{c}\text { Evaluación } \\
\text { escrita }\end{array}$ & Insuficiente & Aceptable & Bueno & $\begin{array}{c}\text { Muy } \\
\text { bueno }\end{array}$ & Excelente \\
\hline
\end{tabular}

- Cuestionario para la entrevista semiestructurada y registración: basada sobre tres preguntas abiertas al sujeto sobre la profesión.

Esta entrevista es directa entre la docente psicomotricista que entrevista y el sujeto entrevistado para recepcionar informaciones relevantes sobre la profesión y conocimientos sobre la formación personal

\section{UNIBE REVISTA}


desarrollada.

La presentación de los resultados se da a través de la descripción de los casos, utilizando las mismas palabras de los entrevistados. El objetivo no es alterar los datos recopilados tratando de trasmitir la inmediatez de la situación presentada. Para los sujetos entrevistados la entrevista se desarrolla en idiomas locales.

- Diseño de la figura humana: los datos de evaluación del diseño de la figura humana son basados en el Test di K. Machover ${ }^{8}, 1949$, utilizado para la exploración de la personalidad. A través de cuerpo se manifiesta lo que uno es y lo que uno quiere ser. El diseño de la figura humana es una proyección de la imagen corporal que pertenece al sujeto que dibuja a nivel simbólico, funcional y afectivo relacional. Figura $=$ Imagen de sí mismo.

\section{METODOLOGÍA}

\section{Investigación explorativa etnográfica}

En cuanto a la finalidad de una investigación sea a nivel cuantitativo o cualitativo, nos encontramos en el campo de acción principalmente para conocer. La frustración del psicomotricista en su trabajo, nace de la necesidad de entender lo que observa a nivel corporal, y saber deducir el significado de las acciones, cogniciones, comunicaciones y emociones.

Para ser sincera, en cuanto a la idea de investigación inicial, he realizado cambios de contenidos, para no trascribir los datos culturales de mi país evidenciados en la identidad cultural.

En cuanto a lo que se refiere al problema de objetividad, soy consciente de que es posible mantener con cierta facilidad en destaque el objeto de investigación en las ciencias exactas, pero lastimosamente no es así en las ciencias antropológicas y humanas como la psicomotricidad, donde se relaciona con seres humanos. En este último caso una pura objetividad la considero improbable, pero no imposible.

\section{Fases de la investigación}

Desde el inicio, la investigación se basa en una meticulosa investigación bibliográfica necesaria para definir el complejo contexto de investigación referente a los datos relativos a contenidos de cultura e identidad guaraní

${ }^{8}$ Machover Karen, Psicóloga Americana (1910-1996). 
el "ñande reko" que hoy día es presente en el paraguayo. Es de relevancia dar a conocer mi involucramiento personal como paraguaya y a la vez como docente en algunos encuentros de formación personal con los estudiantes psicomotricistas en la UNIBERO así como en el CISERPP. Desde hace más de cinco años que a través de una continua observación me ha facilitado instrumentos útiles y valiosos para realizar esta investigación.

\section{Recopilación de datos}

Es un hermoso desafío, no fácil, específicamente me refiero al grupo de Paraguay por el contexto donde se desarrolla la investigación. En esta "no facilidad" no me refiero a la falta de disponibilidad de los participantes, sino a la escasa disponibilidad de tiempo en el territorio. Mi sucesiva presencia a lo largo del tiempo me ha facilitado en los últimos años tomar conciencia sobre la diversidad cultural en términos de identidad de base respecto al segundo grupo. A mi parecer es importante analizar para poder trabajar con los demás con respeto, sin prejuicios, para crear la empatía, que es la herramienta vencedora de cada psicomotricista.

En cuanto al grupo de Italia, debo reconocer que fue mucho más fácil desde el punto de vista de la disponibilidad temporo-espacial. Mi presencia como docente en algunos encuentros ha madurado la confianza y la disponibilidad, de parte de los responsables y alumnos, para realizar el trabajo de investigación sin ninguna dificultad.

\section{Análisis de datos}

El análisis de los datos recopilados en cuanto a la observación requiere una acertada y objetiva evaluación de los parámetros de cada participante. Fue un trabajo largo y cansador, ha requerido tiempo, por la complejidad del planteamiento del problema.

\section{Correlación de datos entre ambos países}

En cuanto a la correlación de los datos de ambos países (Paraguay-Italia), a mi parecer no fue del todo necesaria siendo una investigación de tipo explorativo-cualitativo, no obstante se pudo demostrar la correspondencia de los datos recogidos, asignando un puntaje (escrito y numérico) a cada parámetro evaluado. Evidenciar algunos parámetros mayormente relevantes que irán a tocar datos culturales en contextos diferentes.

\section{Presentación de los resultados}

Se describen en el presente trabajo los resultados de los datos mayormente relevantes para dar respuestas a las preguntas planteadas inicialmente.

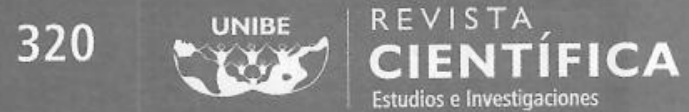




\section{RESULTADOS DE LA INVESTIGACIÓN}

Serán presentados como resultados algunos puntos mayormente evidenciados. En cuanto al primer punto que se refiere al aspecto cultural del Paraguay, sobre el "paradigma chamánico". Durante el proceso de formación corporal, en las observaciones de los parámetros de interacción corporal en la identidad de base, en los estudiantes de la UNIBERO, ¿Subsisten la influencia del paradigma chamánico? ¿Cómo emergen en los estudiantes durante el proceso de formación corporal en psicomotricidad?

En una óptica de respeto hacia diferentes modelos culturales, dando vida a una cultura de pertenencia abierta y móvil, capaz de una proyección hacia las diferencias, desafío constante de los procesos formativos, resulta evidente la existencia de este paradigma complejo y rico de significados. En efecto inspira en una intercultura igualitaria y al plural que da cuerpo a una integración sin asimilación en un mundo globalizado.

En cuanto a mis competencias profesionales como psicomotricista, acentuaré diferentes puntos que engloban las dimensiones de la persona sin perder de vista el modelo cultural.

En una dimensión motriz, sabemos que la motricidad encuentra fundamentalmente su expresión en los múltiples esquemas motores coordinados por el cerebro, que requiere la sincronización de una variedad de informaciones, experiencias sensoriales y motoras en relación con el espacio que circunda el cuerpo. Los datos que emergen en los participantes en esta dimensión de acuerdo a los parámetros evaluados singularmente son: en cuanto al movimiento, elemento esencial en psicomotricidad, son tendencialmente movimientos específicos, intencionales, con poca amplitud demostrando la inhibición para no invadir el espacio de los demás; cada uno realiza la acción según su propio ritmo y tiempo, donde el cuerpo es el centro de las acciones, todos los participantes descalzos proyectando el contacto con la tierra, igual que los antepasados guaraníes, que respetaban la tierra madre como fuente de riqueza y donación para la sobrevivencia.

En cuanto a la utilización del espacio que es una función psicomotriz de base, constituye un cuadro de referencia para el aprendizaje y la adaptación al contexto. Los participantes se mueven libremente utilizando la sala disponible para la actividad, donde el espacio personal aumenta mayormente respecto a la distancia estándar $(40 \mathrm{~cm}-1,20 \mathrm{~m})$ 
el de los participantes eran $60 \mathrm{~cm}-1,60 \mathrm{~m}$. Esto es debido al uso de la dimensión espacial psíquica que es proyectada inconscientemente en nuestro espacio físico.

La utilización del tiempo. Bien sabemos que el tiempo presenta una valencia subjetiva que es considerada en psicomotricidad. En la actividad desarrollada, cada participante vive su tiempo en la ejecución de las acciones, un factor destacable es la relación que cada uno presenta a nivel cronológico; es decir la llegada después de la hora establecida con un mínimo de $30 \mathrm{~min}$. de retraso. Es un factor cultural subrayado en cada actividad realizada fuera o dentro del ámbito formativo. Propio de los guaraníes que viven el tiempo presente, sin apuros, en donde miden la noción del tiempo a través del "Kuarahy" ${ }^{9}$, elemento indispensable para medir el día y la noche.

En la coordinación general, se evidencia una buena destreza y armonía de los movimientos, acorde a las actividades requeridas.

En el plano afectivo-relacional, es evidente la dificultad de expresar las emociones, especialmente aquellas negativas. En algunos momentos, resultan enmascaradas algunas emociones, en cambio, en otros momentos aparecen en modo exagerado (risas insistentes), en donde tendencialmente se tapan la boca, una reacción inmediata, ya sea por vergüenza o miedo de ser criticados. En este plano también resalta la falta de valorización de los objetos, la capacidad de servicio, con una fácil disponibilidad hacia los demás.

Gracias a mi experiencia personal, he evidenciado que el saludo entre amigos y conocidos se da a través de besos en las mejillas; en este aspecto, el beso presenta su lateralidad, es decir desde la mejilla derecha hacia la mejilla izquierda en relación con los italianos, que se da en modo contrario, es decir, se inicia en la mejilla izquierda hacia la mejilla derecha.

En el plano comunicativo, a nivel verbal se evidencia en la mayoría una relación ambivalente con el idioma: por un lado, el castellano (idioma introducido por los colonizadores en época de la conquista) y el guaraní (idioma de los primeros habitantes del Paraguay). Este último reconocido como idioma oficial del país. En la funcionalidad del idioma se da una mezcla de ambos idiomas, dando origen al "castellano paraguayo". Durante la actividad de formación corporal la gran mayoría hablaban en

${ }^{9}$ Kuarahy, vocablo guaraní que indica Sol.

\section{2}


castellano, debido al contexto educativo donde se desarrolla la acción: la escuela es un espacio de educación formal que nació en época colonial, relacionado con el "saber occidental".

Otro punto que es importante describir es la dificultad de verbalizar las emociones vivenciadas de parte de los participantes. Tendencialmente verbalizan entre sí en voz baja durante la realización del trabajo corporal.

Es mucho más fácil insertarse en el mundo de la comunicación no verbal, donde el cuerpo es el mediador de las comunicaciones.

En el plano cognitivo, la memoria en cuanto propiedad de conservar algunas informaciones contenidas en un mensaje precedentemente vivenciado, en los participantes aparecen dificultosas, especialmente aquellas de fijación y consolidación en el tiempo. La memoria corpórea vivenciada a través del tono en las diferentes actividades es rica de significados, especialmente en el momento de la relajación donde los participantes han vivido como espacio de distensión y de placer.

En cuanto a los diferentes espacios de organizaciones, ya sea temporoespacial o las mismas actividades a través del movimiento, aparece mayormente una valencia funcional antes que afectivo relacional, en donde las acciones son vivenciadas en el hacer más bien en el sentir.

Es curiosa la modalidad de relacionarse con el cuerpo, que a mi parecer presenta un bagaje cultural rico de significados, como por ejemplo la modalidad de contar los números a través de los dedos muy diferente a los pares italianos. En Paraguay comenzamos a contar con el dedo meñique para terminar con el pulgar; en cambio, en Italia se utiliza la secuencialidad de los dedos, es decir se inicia con el pulgar y se termina con el meñique.

En el plano comportamental, en este ítem es importante subrayar la capacidad de socialización y amistad que presentan los participantes entre sí. Demuestran espíritu de adaptabilidad a las situaciones, como por ejemplo, por un lado, el hecho de esperar a los demás para comenzar la actividad no obstante 30 minutos de retraso, sin incomodarse (hora paraguaya). Por otro lado, el hecho de adaptarse a los materiales presentados para la actividad con disponibilidad y creatividad.

Otro aspecto resaltante es la dificultad en algunos de iniciar la actividad en primera persona, la necesidad de observar o realizar determinada acción en compaña. En cuantos a las actividades realizadas, es bueno 
destacar la capacidad de terminar las acciones realizadas con espíritu de alegría y adaptabilidad.

Observando el comportamiento, una acción que me parece importante describir como factor cultural en los sujetos es el modo en que la gran mayoría han trasportado sus carteras o bolsones, apoyándolas sobre el hombro izquierdo respecto a sus pares italianos que trasportan apoyándolas hacía el hombro derecho.

-Respecto a la segunda pregunta, utilizando la misma actividad de expresión corporal con los estudiantes del CISERPP y de la UNIBERO en la correlación de los parámetros psicomotores evaluados, ¿Existen diferencias significativas entre ambos países?

La formación corporal, más allá del contexto cultural, ofrece al sujeto la posibilidad de relacionarse consigo mismo y con los demás, en poder reflexionar sobre sus acciones, sus pensamientos y sus emociones. Bien sabemos que datos corporales presentan lenguajes universales, que al mismo tiempo enriquecen y diferencian a las personas de un determinado contexto.

-En la primera tabla se presentan datos de evaluación a nivel cuantitativo que presentan cada sujeto de CISERPP como puntaje desde 5 hasta 10 en los parámetros de interacción corporal. Se puede evidenciar mínimas diferencias entre un sujeto y otro. Las diferencias significativas se notan en el puntaje de los sujetos C2 e C3 que han presentado el máximo respecto a los demás, es debido al grado de formación, es decir, frecuentan el último año, por ende, han madurado y se han familiarizado con las técnicas adoptadas en una formación corporal. La media de los puntajes generales son de: 7.6 que corresponde a bueno.

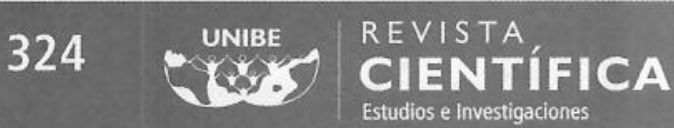


Tabla 1 - Parámetros de interacción corporal

\begin{tabular}{|c|c|c|c|c|c|c|c|c|c|}
\hline Sujetos Ciserpp & C1 & $\mathrm{C} 2$ & C3 & C4 & C5 & C6 & C7 & C8 & C9 \\
\hline Aspecto general & 9 & 9 & 9 & 8 & 8 & 9 & 8 & 8 & 8 \\
\hline Actividad espontánea & 8 & 9 & 9 & 8 & 8 & 8 & 7 & 7 & 7 \\
\hline Motricidad & 7 & 8 & 9 & 8 & 7 & 8 & 7 & 7 & 7 \\
\hline Tono & 7 & 8 & 9 & 8 & 8 & 8 & 8 & 7 & 7 \\
\hline Postura & 8 & 9 & 9 & 8 & 8 & 8 & 8 & 8 & 8 \\
\hline Equilibrio & 8 & 9 & 9 & 7 & 7 & 8 & 7 & 7 & 8 \\
\hline Control emocional & 7 & 9 & 9 & 7 & 8 & 8 & 8 & 8 & 8 \\
\hline Ritmo & 7 & 9 & 9 & 8 & 7 & 7 & 7 & 8 & 7 \\
\hline Lateralidad & 1 & 1 & 1 & 1 & 1 & 3 & 1 & 1 & 1 \\
\hline Espacio & 8 & 9 & 9 & 9 & 8 & 8 & 8 & 8 & 8 \\
\hline Tiempo & 8 & 9 & 9 & 8 & 8 & 8 & 8 & 8 & 8 \\
\hline Esquema corporal & 7 & 9 & 9 & 8 & 8 & 7 & 8 & 8 & 7 \\
\hline Praxis & 8 & 9 & 9 & 8 & 7 & 7 & 7 & 7 & 7 \\
\hline Objeto & 8 & 9 & 9 & 8 & 8 & 7 & 8 & 8 & 7 \\
\hline Control visiva & 8 & 8 & 9 & 7 & 8 & 8 & 8 & 8 & 8 \\
\hline Control auditiva & 8 & 8 & 9 & 8 & 8 & 8 & 8 & 8 & 8 \\
\hline Memoria & 8 & 8 & 9 & 7 & 8 & 8 & 8 & 8 & 8 \\
\hline $\begin{array}{l}\text { Capacidad de atención } \\
\text { general }\end{array}$ & 7 & 8 & 9 & 7 & 7 & 9 & 8 & 7 & 8 \\
\hline Comunicación & 8 & 9 & 9 & 8 & 8 & 8 & 8 & 8 & 8 \\
\hline $\begin{array}{l}\text { Aspectos } \\
\text { Comporzzztamentales }\end{array}$ & 8 & 9 & 9 & 8 & 8 & 8 & 8 & 8 & 8 \\
\hline
\end{tabular}

Leyenda: Lateralidad: 1 = derecha; 2 = izquierda; 3 = ambas 
En la segunda tabla se presentan datos de evaluación a nivel cuantitativo de cada participante de la UNIBERO como puntaje desde 5 hasta 10 en los parámetros de interacción corporal. Se puede evidenciar mínimas diferencias entre un sujeto y otro. Es debido a la escasa familiaridad con la técnica utilizada y también relacionadas a datos culturales. La media general es de: 7,45 que corresponde a discreto.

Tabla 2 - Parámetros de interacción corporal

\begin{tabular}{|c|c|c|c|c|c|c|c|}
\hline Sujetos UNIBERO & I1 & I2 & I3 & I4 & I5 & I6 & I7 \\
\hline Aspecto generales & 8 & 9 & 8 & 8 & 9 & 8 & 8 \\
\hline Actividad espontánea & 7 & 9 & 8 & 8 & 8 & 7 & 8 \\
\hline Motricidad & & 8 & 8 & 8 & 8 & 7 & 8 \\
\hline Tono & 7 & 9 & 8 & 7 & 8 & 7 & 8 \\
\hline Postura & 8 & 8 & 8 & 8 & 8 & 8 & 8 \\
\hline Equilibrio & 8 & 8 & 7 & 7 & 9 & 7 & 8 \\
\hline Control emocional & 8 & 8 & 7 & 8 & 8 & 7 & 9 \\
\hline Ritmo & 7 & 7 & 7 & 7 & 8 & 7 & 9 \\
\hline Lateralidad & 1 & 1 & 3 & 1 & 1 & 1 & 1 \\
\hline Espacio & 8 & 8 & 8 & 8 & 8 & 8 & 8 \\
\hline Tiempo & 8 & 7 & 7 & 7 & 8 & 7 & 8 \\
\hline Esquema corporal & 7 & 8 & 7 & 8 & 8 & 8 & 8 \\
\hline Praxia & 7 & 8 & 8 & 7 & 7 & 7 & 8 \\
\hline Objeto & 8 & 8 & 8 & 8 & 7 & 7 & 8 \\
\hline Control visiva & 8 & 7 & 8 & 8 & 8 & 8 & 8 \\
\hline Control auditiva & 8 & 8 & 8 & 8 & 8 & 8 & 8 \\
\hline Memoria & 7 & 7 & 7 & 8 & 8 & 8 & 7 \\
\hline Capacidad de atención general & 7 & 8 & 7 & 8 & 7 & 8 & 7 \\
\hline Comunicación & 8 & 8 & 8 & 8 & 8 & 8 & 8 \\
\hline Aspecto comportamental & 8 & 8 & 8 & 8 & 8 & 8 & 8 \\
\hline
\end{tabular}

Leyenda: Lateralidad: 1 = derecha; 2 = izquierda; 3 = ambas

\section{UNIBE REVISTA


Las últimas tablas comparativas, realizadas gracias a la colaboración del docente Sartori R. ${ }^{10}$ se puede observar, que en la correlación de los datos entre los participantes de ambos países existen diferencias mínimas a excepción en dos parámetros como: tempo y memoria. Debidos a los datos culturales precedentemente presentados.

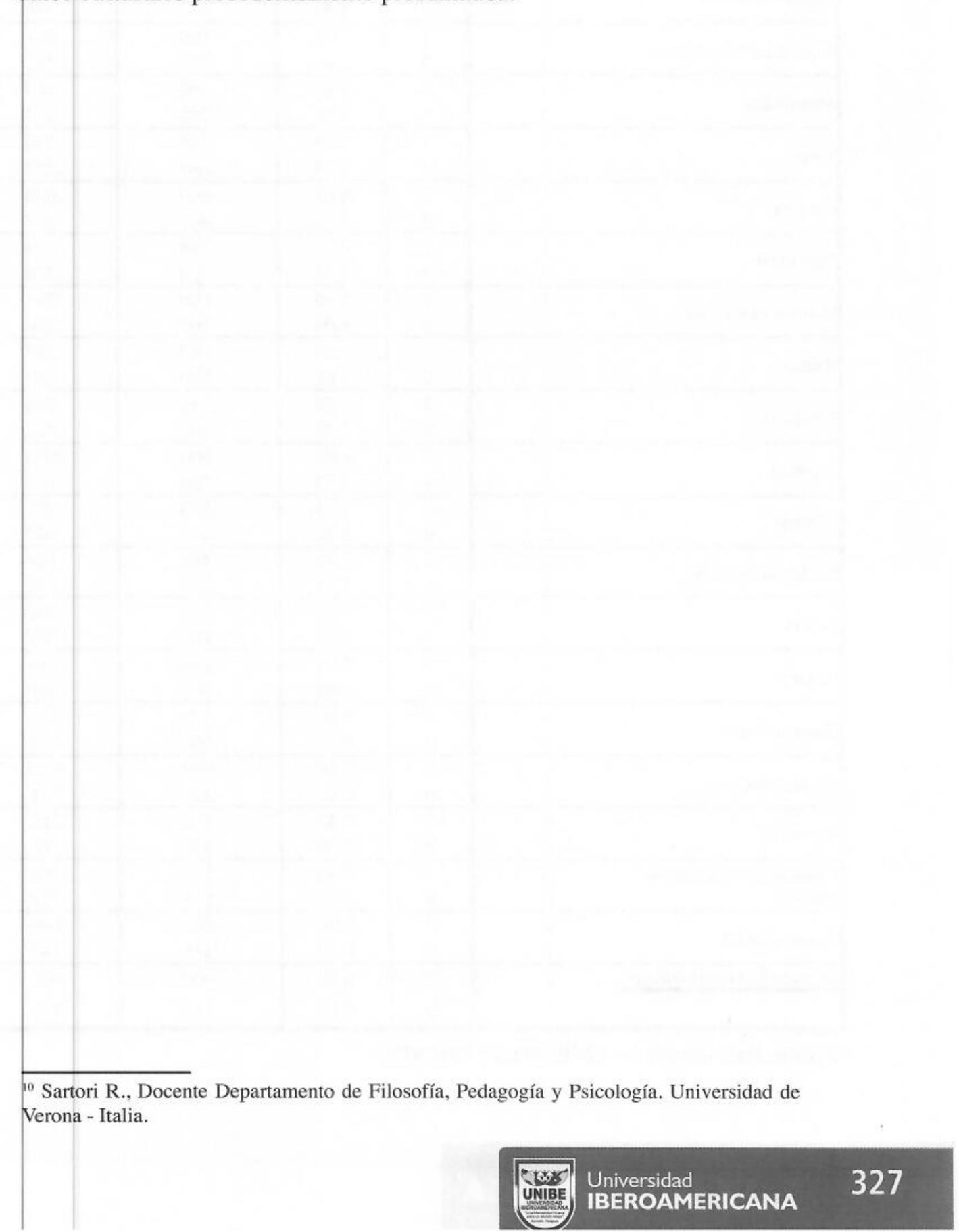


Tabla 3 - Estadísticas de ambos grupos

\begin{tabular}{|c|c|c|c|c|c|}
\hline & Proveniencia & $\mathbf{N}$ & Media & Desviación std. & $\begin{array}{l}\text { Error std. } \\
\text { Media }\end{array}$ \\
\hline \multirow{2}{*}{ Aspecto general } & 1 & 7 & 8.29 & .488 & .184 \\
\hline & 2 & 9 & 8.44 & .527 & .176 \\
\hline \multirow{2}{*}{ Actividad espontánea } & 1 & 7 & 7.86 & .690 & .261 \\
\hline & 2 & 9 & 7.89 & .782 & .261 \\
\hline \multirow{2}{*}{ Motricidad } & 1 & 6 & 7.83 & .408 & .167 \\
\hline & 2 & 9 & 7.56 & .726 & .242 \\
\hline \multirow{2}{*}{ Tono } & 1 & 7 & 7.71 & .756 & .286 \\
\hline & 2 & 9 & 7.78 & .667 & .222 \\
\hline \multirow{2}{*}{ Postura } & 1 & 7 & 8.00 & .000 & .000 \\
\hline & 2 & 9 & 8.22 & .441 & .147 \\
\hline \multirow{2}{*}{ Equilibrio } & 1 & 7 & 7.71 & .756 & .286 \\
\hline & 2 & 9 & 7.78 & .833 & .278 \\
\hline \multirow{2}{*}{ Control emocional } & 1 & 7 & 7.86 & .690 & .261 \\
\hline & 2 & 9 & 8.00 & .707 & .236 \\
\hline \multirow{2}{*}{ Ritmo } & 1 & 7 & 7.43 & .787 & .297 \\
\hline & 2 & 9 & 7.67 & .866 & .289 \\
\hline \multirow{2}{*}{ Lateralidad } & 1 & 7 & 1.29 & .756 & .286 \\
\hline & 2 & 9 & 1.22 & .667 & .222 \\
\hline \multirow{2}{*}{ Espacio } & 1 & 7 & 8.00 & .000 & .000 \\
\hline & 2 & 9 & 8.33 & .500 & .167 \\
\hline \multirow{2}{*}{ Tiempo } & 1 & 7 & 7.43 & .535 & .202 \\
\hline & 2 & 9 & 8.22 & .441 & .147 \\
\hline \multirow{2}{*}{ Esquema corporal } & 1 & 7 & 7.71 & .488 & .184 \\
\hline & 2 & 9 & 7.78 & .833 & .278 \\
\hline \multirow{2}{*}{ Praxia } & 1 & 7 & 7.43 & .535 & .202 \\
\hline & 2 & 9 & 7.67 & .866 & .289 \\
\hline \multirow{2}{*}{ Objeto } & 1 & 7 & 7.71 & .488 & .184 \\
\hline & 2 & 9 & 8.00 & .707 & .236 \\
\hline \multirow{2}{*}{ Control visiva } & 1 & 7 & 7.86 & .378 & .143 \\
\hline & 2 & 9 & 8.00 & .500 & .167 \\
\hline \multirow{2}{*}{ Control auditiva } & 1 & 7 & 8.00 & .000 & .000 \\
\hline & 2 & 9 & 8.11 & .333 & .111 \\
\hline \multirow{2}{*}{ Memoria } & 1 & 7 & 7.43 & .535 & .202 \\
\hline & 2 & 9 & 8.00 & .500 & .167 \\
\hline \multirow{2}{*}{$\begin{array}{l}\text { Capacidad de atención } \\
\text { general }\end{array}$} & 1 & 7 & 7.43 & .535 & .202 \\
\hline & 2 & 9 & 7.78 & .833 & .278 \\
\hline \multirow{2}{*}{ Comunicación } & 1 & 7 & 8.00 & .000 & .000 \\
\hline & 2 & 9 & 8.22 & .441 & .147 \\
\hline \multirow[t]{2}{*}{ Aspecto Comportamental } & 1 & 7 & 8.00 & .000 & .000 \\
\hline & 2 & 9 & 8.22 & .441 & .147 \\
\hline
\end{tabular}

Leyenda: Proveniencia: 1 = UNIBERO; 2 = CISERPP

\section{UNIBE ${ }^{\text {REVISTA }}$ CIENTÍFICA}


Tabla 4 - Test por campeones independientes

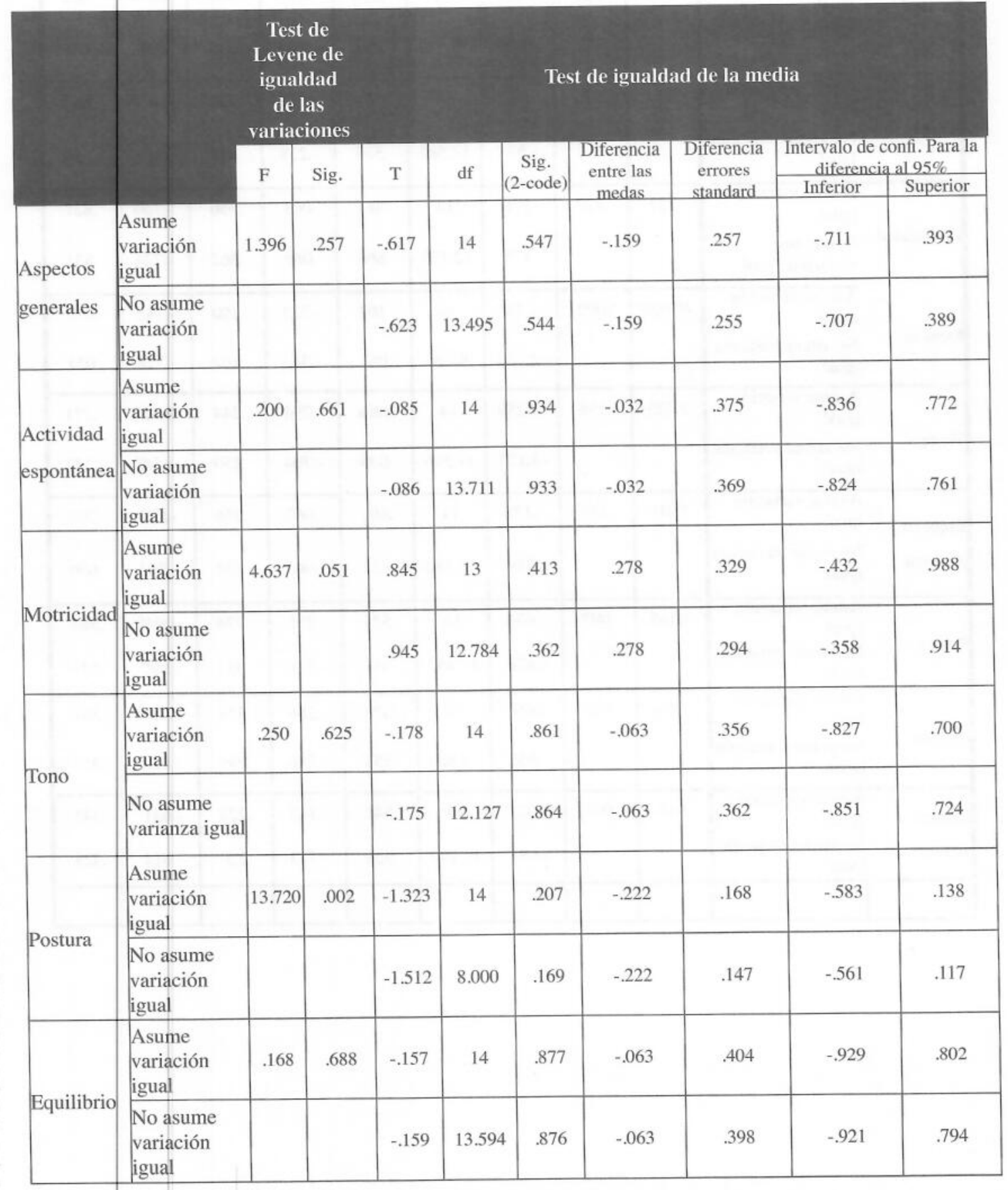




\begin{tabular}{|c|c|c|c|c|c|c|c|c|c|c|}
\hline $\begin{array}{l}\text { Control } \\
\text { emocional }\end{array}$ & $\begin{array}{l}\text { Asume variación } \\
\text { igual } \\
\text { No asume variación } \\
\text { igual }\end{array}$ & .033 & .858 & $\begin{array}{l}-.405 \\
-.406\end{array}$ & $\begin{array}{c}14 \\
13.199\end{array}$ & $\begin{array}{l}.692 \\
.691\end{array}$ & $\begin{array}{l}-.143 \\
-.143\end{array}$ & $\begin{array}{l}.353 \\
.352\end{array}$ & $\begin{array}{l}-.899 \\
-.901\end{array}$ & $\begin{array}{l}.614 \\
.615\end{array}$ \\
\hline Ritmo & $\begin{array}{l}\text { Asume variación } \\
\text { igual } \\
\text { No asume variación } \\
\text { igual }\end{array}$ & .423 & .526 & $\begin{array}{l}-.567 \\
-.574\end{array}$ & $\begin{array}{c}14 \\
13.587\end{array}$ & $\begin{array}{l}.580 \\
.575\end{array}$ & $\begin{array}{l}-.238 \\
-.238\end{array}$ & $\begin{array}{l}.420 \\
.414\end{array}$ & $\begin{array}{l}-1.138 \\
-1.130\end{array}$ & $\begin{array}{l}.662 \\
.653\end{array}$ \\
\hline Lateralidad & $\begin{array}{l}\text { Asume variación } \\
\text { igual } \\
\text { Non asume } \\
\text { variación igual } \\
\end{array}$ & .127 & .727 & $\begin{array}{l}.178 \\
.175\end{array}$ & $\begin{array}{c}14 \\
12.127\end{array}$ & $\begin{array}{l}.861 \\
.864\end{array}$ & $\begin{array}{l}.063 \\
.063\end{array}$ & $\begin{array}{l}.356 \\
.362\end{array}$ & $\begin{array}{l}-.700 \\
-.724\end{array}$ & $\begin{array}{l}.827 \\
.851\end{array}$ \\
\hline Espacio & $\begin{array}{l}\text { Asume variación } \\
\text { igual } \\
\text { No asume varianza } \\
\text { igual }\end{array}$ & 49.000 & .000 & $\begin{array}{l}-1.750 \\
-2.000\end{array}$ & $\begin{array}{c}14 \\
8.000\end{array}$ & $\begin{array}{l}.102 \\
.081\end{array}$ & $\begin{array}{l}-.333 \\
-.333\end{array}$ & $\begin{array}{l}.190 \\
.167\end{array}$ & $\begin{array}{l}-.742 \\
-.718\end{array}$ & $\begin{array}{l}.075 \\
.051\end{array}$ \\
\hline Tiempo & $\begin{array}{l}\text { Asume variación } \\
\text { igual } \\
\text { No asume variación } \\
\text { igual }\end{array}$ & 2.223 & .158 & $\begin{array}{l}-3.259 \\
-3.177\end{array}$ & $\begin{array}{c}14 \\
11.596\end{array}$ & $\begin{array}{l}.006 \\
.008\end{array}$ & $\begin{array}{l}-.794 \\
-.794\end{array}$ & $\begin{array}{l}.244 \\
.250\end{array}$ & $\begin{array}{l}-1.316 \\
-1.340\end{array}$ & $\begin{array}{l}-.271 \\
-.247\end{array}$ \\
\hline $\begin{array}{l}\text { Esquema } \\
\text { corporal }\end{array}$ & $\begin{array}{l}\text { Asume variación } \\
\text { igual } \\
\text { No asume variación } \\
\text { igual }\end{array}$ & 2.916 & .110 & $\begin{array}{l}-.178 \\
-.190\end{array}$ & $\begin{array}{c}14 \\
13.190\end{array}$ & .852 & $\begin{array}{l}-.063 \\
-.063\end{array}$ & $\begin{array}{l}.356 \\
.333\end{array}$ & $\begin{array}{l}-.827 \\
-.783\end{array}$ & $\begin{array}{l}.700 \\
.656\end{array}$ \\
\hline Praxia & $\begin{array}{l}\text { Asume variación } \\
\text { igual } \\
\text { No asume varianza } \\
\text { igual } \\
\end{array}$ & 3.165 & .097 & $\begin{array}{l}-.636 \\
-.676 \\
\end{array}$ & $\begin{array}{c}14 \\
13.453\end{array}$ & $\begin{array}{l}.535 \\
.511\end{array}$ & $\begin{array}{l}-.238 \\
-.238\end{array}$ & $\begin{array}{l}.374 \\
.352\end{array}$ & $\begin{array}{l}-1.040 \\
-.997\end{array}$ & $\begin{array}{l}.564 \\
.521\end{array}$ \\
\hline Oggetti & $\begin{array}{l}\text { Asume variación } \\
\text { igual } \\
\text { No asume variación } \\
\text { igual }\end{array}$ & .029 & .867 & $\begin{array}{l}-.910 \\
-.955\end{array}$ & $\begin{array}{c}14 \\
13.865\end{array}$ & $\begin{array}{l}.378 \\
.356\end{array}$ & $\begin{array}{l}-.286 \\
-.286\end{array}$ & $\begin{array}{l}.314 \\
.299\end{array}$ & $\begin{array}{l}-.959 \\
-.928\end{array}$ & $\begin{array}{l}.387 \\
.357\end{array}$ \\
\hline $\begin{array}{l}\text { Control } \\
\text { visiva }\end{array}$ & $\begin{array}{l}\text { Asume variación } \\
\text { igual } \\
\text { No asume variación } \\
\text { igual }\end{array}$ & .014 & .907 & $\begin{array}{l}-.627 \\
-.651\end{array}$ & \begin{tabular}{c|}
14 \\
13.999
\end{tabular} & $\begin{array}{l}.540 \\
.526\end{array}$ & $\begin{array}{l}-.143 \\
-.143\end{array}$ & $\begin{array}{l}.228 \\
.220\end{array}$ & $\begin{array}{l}-.631 \\
-.614\end{array}$ & $\begin{array}{l}.345 \\
.328\end{array}$ \\
\hline & & & & & & & & & & \\
\hline
\end{tabular}

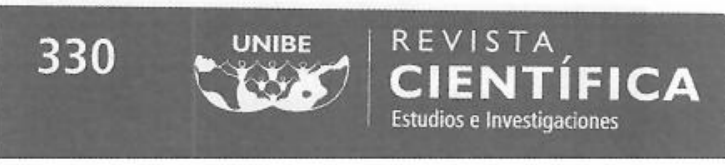




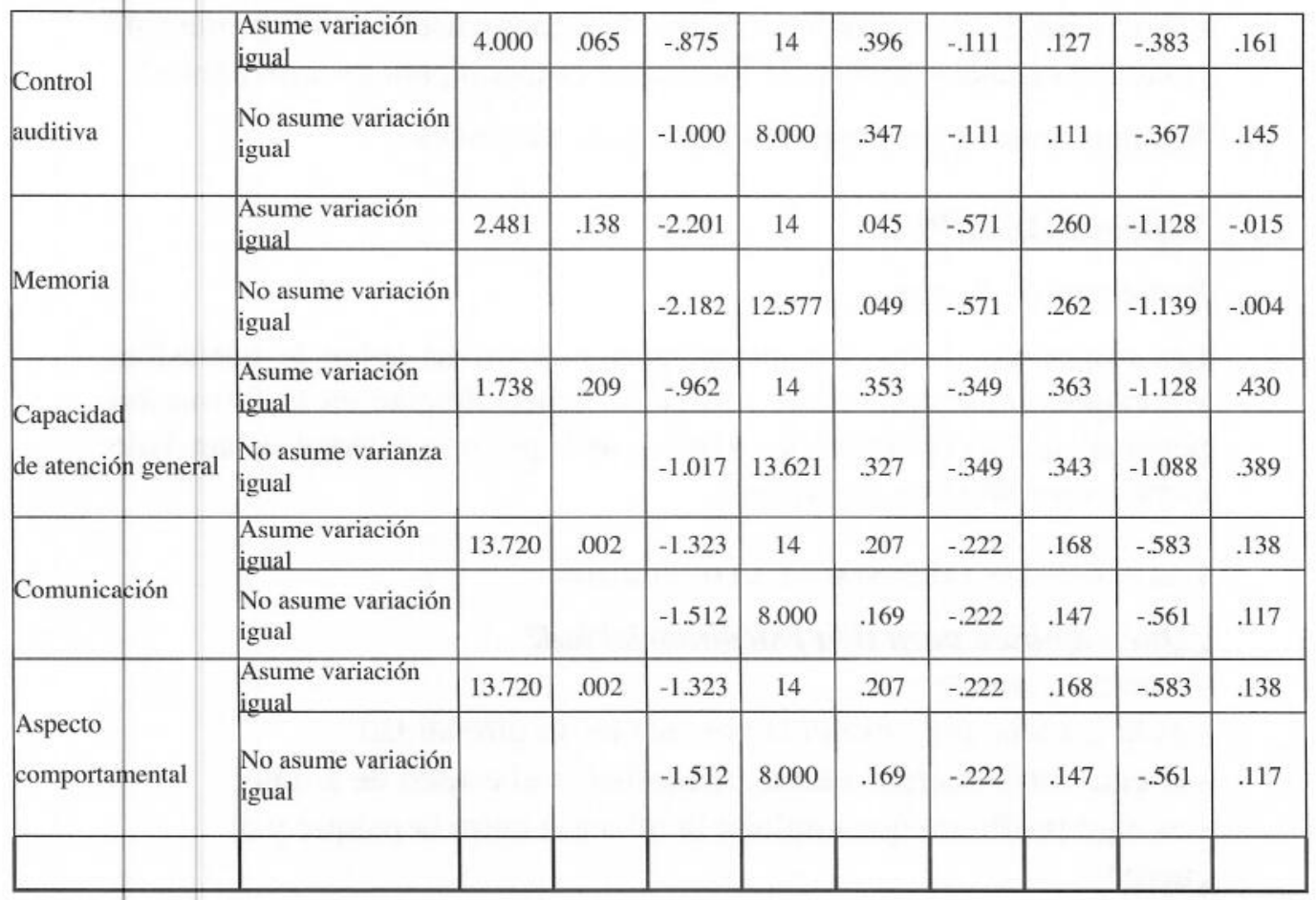


En relación a la entrevista realizada a los sujetos sobre: ¿Qué conocimientos presentan los alumnos de ambos países sobre la profesión? ¿Qué importancia presenta la formación corporal para los estudiantes?

Seguidamente se presentan los datos más relevantes.

\section{Sujetos: CISERPP}

Participantes: 9 sujetos.

Las respuestas dadas son interesantes y positivas sobre la profesión, valorizando el cuerpo como mediador, especialmente en la formación personal, todos confirman que a través de la psicomotricidad se han dado cambios positivos en sus vidas.

\section{LAS PREGUSTAS PRESENTADAS A LOS SUJETOS}

\section{¿Qué significa para ti la psicomotricidad?}

- Cuerpo y mente;

- es la ciencia que estudia la persona en su globalidad;

- el equilibrio cuerpo -mente, reequilibrar el estado de ánimo;

- es algo profundo que engloba la relación entre la psique y el cuerpo;

- trabajar el estado emocional de las personas;

- es difícil de explicar es mejor vivirla;

- es una disciplina que trabaja la persona como unidad;

- bienestar cuerpo-mente;

- descubrir la diversidad.

¿Qué es para ti la formación corporal en psicomotricidad?

- Es un leer el cuerpo, es un conjunto de capacidades físicas, psicológicas que el cuerpo puede hacer y no hacer dictada por las emociones a través del pensamiento;

- es la manifestación externa del bienestar de la persona;

- es fundamental porque nos exprimimos a través del cuerpo;

- ponemos en juego nosotros mismos, nuestras acciones y

pensamientos para después compartir con otra persona;

- es una búsqueda de relación personal con el otro;

- aprender algo de tu cuerpo en primera persona;

- es un modo de expresar, a través del cuerpo y el tono en especial;

- seguramente es el pilar fundamental de la psicomotricidad, que es difícil entender por su complejidad;

\section{UNIBE REVISTA


- es un saber trabajar con el cuerpo, hacer ver la propia naturaleza.

¿Cómo te sientes después de un trabajo corporal?

- Muy bien es encontrarse consigo mismo;

- ¡excelente! Es un proceso personal;

bien, para entender al otro;

- muy bien es un descubrirse;

- mi cuerpo tira fuera lo que lleva dentro, jexcelente!;

- súper bien, ¡me encanta!

\section{Sujetos: UNIBERO}

Han participado en la entrevista tres sujetos, que han dado respuestas positivas y significativas sobre la profesión. Valorizando la figura del psicomotricista y la importancia de la implementación de la formación corporal en psicomotricidad y en sus vidas.

\section{APLiCACIONES PRESENTADAS a LOS ESTUdianteS}

¿Qué significa para ti la psicomotricidad?

Es la ciencia que estudia la globalidad de la persona;

- es emoción pura;

- es la ciencia de las relaciones personales.

¿Qué es para ti la formación corporal en psicomotricidad?

-Es algo demasiado importante, es donde nosotros podemos ir formándonos como persona, desarrollarnos como persona y viendo cuáles son nuestras capacidades, nuestras actitudes y hasta qué punto podemos ser nosotros mismos;

- sí, es importante porque al conocer mi cuerpo, puedo trabajar mi cuerpo, yo sé que más adelante voy a poder conocer y entender el cuerpo de la otra persona;

- para mí es esencial para poder entender al otro, para poder relacionarme con él; es todo lo que se pueda expresar a través del cuerpo y con el cuerpo.

¿Cómo te sientes después de un trabajo corporal? 
- Súper bien porque dentro de todo uno se siente relajado, me está costando, entonces uno va conociéndose más a sí mismo;

- siento una tranquilidad, me siento más distendida;

- me sentí súper relajada, bien tranquila, siento que sí me pude expresar que he podido expresar emociones.

- Siendo la identidad parte integrante de la persona, ¿Qué datos emergen durante el proceso de formación corporal en la representación del diseño de la figura humana?

Es interesante, la capacidad de proyección que presentamos los seres humanos, en línea general. Hoy en día utilizamos en psicomotricidad el diseño de la figura humana dándole una connotación dinámica en cuanto al aspecto relacional con el propio cuerpo: imagen corporal y esquema corporal. Es interesante subrayar por una parte que algunos sujetos de CISERPP no han realizado el diseño de la figura humana sustituyendo con otros diseños como: paisajes u objetos. Por otro lado, los demás que han diseñado han dado un toque personal en donde se ven proyectadas sus emociones y acciones.

Los sujetos de la UNIBERO, todos han diseñado dando un toque especial a sus trabajos, como la expresión de sus emociones. Llama la atención que algunos han dado prioridad al diseño de la cabeza y el rostros dejando de lado el cuerpo.

\section{UNIBE REVISTA




\section{CONCLUSIÓN}

En la formación corporal es importante adquirir competencias personales para poder traducir en competencias profesionales capaces de trasmitir y entender: emociones, acciones, cogniciones y comunicaciones a través del cuerpo.

En Paraguay la formación corporal debe permitir reflexionar sobre la riqueza de la cultura y la historia del país, donde el ser y sentir se conjugan en las vivencias personales e interpersonales. La vida humana desde el nacimiento es cuerpo emocional, es comunicación, es intención. No es posible proyectar nuestra vida sin corporeidad tónico-emocional. Es decir, el cuerpo en relación, es cuerpo en movimiento, cuerpo social y cuerpo cultural que es igual a identidad.

Las técnicas de expresión corporal, en psicomotricidad son medios y fines para descubrirse a sí mismo y los demás, es esencial para el bienestar psicocorporal del sujeto.

Creo importante subrayar, a mi parecer indispensable, el aporte de la etnografía en la comprensión de complejas sociedades actuales. Las investigaciones etnográficas hechas en este trabajo en plano explorativo, siendo mínimas han evidenciado en línea general aspectos que caracterizan prácticas culturales de un país en cuanto a formación corporal en psicomotricidad.

Ir a observar detalladamente los parámetros psicomotores de cada sujeto, hace reflexionar sobre la importancia del lenguaje comunicativo del cuerpo, aparentemente comunes y universales para todos, pero que al mismo tiempo únicos y particulares como cada ser humano en su globalidad. La psicomotricidad, atendiendo estos aspectos, valoriza la persona en su individualidad y particularidad.

Por último, aprovecho la ocasión para agradecer profundamente a todos los que han apoyado y colaborado en esta investigación que es la base para impulsar la etnopsicomotricidad en un mundo cada vez más globalizado que descuida las diferencias individuales y sociales del ser humano. 


\section{BIBLIOGRAFÍA}

BISCALDI A. (2004) Our Bad Language. Creolizzazione linguistica e conflitto nell'isola di Antigua, Roma, Cisu.

BOSCAINI F. (2002) Valorizzare le competenze dello psicomotricista, Verona, Res.

BOSCAINI F.; GOBI G. (2001) Il corpo tonico-emozionale. La conoscenza come desiderio in psicomotricità, Verona, Res.

BOSCAINI F. (1992) Iter Psicomotorio, Formazione Professione Persona, Verona, Librería Universitaria.

BOSCAINI F. (2008) L'esame psicomotorio, Verona, Res.

CLASTRES P. (2003) La società contro lo Stato, Verona, Ombre corte.

CORDONA G. R. (1985) I sei lati del mondo, Roma, Laterza.

DURANTI A. (2000) Antropologia Del linguaggio, Roma, Meltemi.

FACCIO E. (2004) Le identità corporee, Milano, Giunti.

GALIMBERTI U.( 2010) Il corpo, Milano, Feltrinelli.

GOBBO F.,TALLE' C (2010), Antropologia ed educazione in America Latina, Roma, Cisu.

GOLEMAN D. (1999) Intelligenza Emotiva, Milano, Rizzoli.

GRUNBERG G.; DE ALMEIDA R.; MELIÁ B. (2000) Ñande reko il nostro modo di essere, Roma, Cisu.

IANES D. (2004) Metacognizione e insegnamento, Trento, Erikson.

KUHN T. (1996) La struttura delle rivoluzioni scientifiche, Torino, Einaudi.

LAPIERRE A. (2001) Dalla psicomotricità relazionale all'analisi corporea della relazione, Roma. Armando.

LELLI S. (2007) Trasformazioni guaranì tra paradigma sciamanico e scuola, Roma, Cisu.

LEWIN K. (1976) I conflitti sociali, Saggi di dinamica di gruppo, Milano, Angeli.

MALESANI P.G. (2009) Deontologia e corporativismi. Chi giudica qualità, competenze e conoscenze dello Psicomotricista? Verona, Res.

\section{UNIBE CIEVISTA $^{\text {REIFICA }}$


MASTROMATTEI R.(2007) “Introduzione”, in Lelli Silvia (a cura di), Trasformazione guaranì tra paradigma sciamanico e scuola, Roma, Cisu. PERMAS J. (2004) Platon y los guaraníes.Asunción, Cepag.

REMOTTI F. (2010) Prima lezione di Antropologia. Bari, Laterza.

ROMERO SANABRIA A.(2007) Más paraguayo que la mandioca. Asunción, El Lector.

ROMERO SANABRIA A. (2009) La autoestima y el paraguayo. Asunción, Il Servilibro.

SCHILDER P. (1988) Immagine di sè e schema corporeo, Milano, Franco Angeli.

SCHULTZ E. A.; LAVENDA R. H.(2001) Antropologia Culturale. Bologna, Zanichelli.

SOUBIRAN G.; COSTE, J.; (1989) Psicomotricidad y relajación psicosomática, Madrid, Gunea.

TELESCA I. (2011) Historia del Paraguay. Santillana, Asunción. 
